\section{Esther von Stebut Kerstin Steinbrink W. Bräuninger}

\title{
Lichen ruber pemphigoides
}

\section{Lichen Planus Pemphigoides}

\section{Zusammenfassung}

Eine 56-jährige Patientin bemerkte zunächst stark juckende Papeln an beiden Unterschenkeln. Im Verlauf weniger Wochen traten zusätzlich prall gespannte Blasen am gesamten Integument auf. Die Probebiopsie einer Papel zeigte histologisch das typische Bild eines Lichen ruber planus mit Hyperparakeratose und nekrotischen Keratinozyten in der Basalzellage mit dichtem lymphozytären Infiltrat. Die Biopsie aus einem bullösen Areal wies eine subepidermale Spaltbildung mit zahlreichen neutrophilen und eosinophilen Granulozyten auf. In der direkten Immunfluoreszenz fanden sich bandförmige $C_{3}$-Ablagerungen $\mathrm{zu}$ beiden Seiten der Spaltbildung. Zirkulierende Antikörper konnten nicht nachgewiesen werden. Aufgrund des klinischen Bildes und des charakteristischen histomorphologischen Befundes einschließlich Immunfluoreszenz konnten wir die Diagnose Lichen ruber pemphigoides stellen. Diese sehr seltene Variante des Lichen ruber zeigt sowohl Merkmale eines Lichen ruber als auch eines bullösen Pemphigoids. Molekularbiologische Befunde weisen neuerdings darauf hin, dass die für den Lichen ruber pemphigoides verantwortlichen Autoantikörper Reaktivität gegen BP180 und ein 200 kDa großes, epidermales Antigen zeigen. Warum nicht bei allen Patienten zirkulierende Autoantikörper nachweisbar sind, ist bisher ungeklärt.

\section{Abstract}

A 56-year-old female patient suffered from multiple pruritic papules on her dorsal legs. Within a few weeks, she additionally developed blisters on her extremities and the trunk. Histology of a papule revealed typical features of lichen planus with hyperparakeratosis, necrotic basal keratinocytes and a dense lymphocytic infiltrate. Another biopsy of a skin lesion with blister showed subepidermal dissociation and multiple neutrophil and eosinophil granulocytes. Direct immunofluorescence displayed linear deposition of complement $\mathrm{C} 3$ at the basal membrane. Circulating antibodies were not detected. From the clinical appearance and the histological findings including immunofluorescence results we diagnosed lichen planus pemphigoides. This rare variant of lichen planus shows features of both lichen planus and bullous pemphigoid. However, recent molecular biological investigations suggested that in patients with lichen planus pemphigoides autoantibodies with reactivity against BP180 and a $200 \mathrm{kDa}$ autoantigen are responsible for disease development. Why, however, in some patients no circulating autoantibodies can be detected will have to be investigated. 


\section{Anamnese}

Eine 56-jährige Patientin wies initial im Bereich beider Schienbeine stark juckende Papeln auf. Im Verlauf der folgenden 4 Wochen breiteten sich die Hautveränderungen auf das gesamte Integument aus. Außerdem zeigte sich eine ausgeprägte Blasenbildung, weswegen die Patientin uns zugewiesen wurde.

\section{Erst-/ Aufnahmebefund}

Die Patientin wirkte zum Zeitpunkt der Erstvorstellung bei reduziertem Allgemeinzustand nervös und klagte über unstillbaren Juckreiz. Im Bereich des gesamten Integumentes mit Betonung der Extremitätenstreckseiten und unter Aussparung des Kopfes zeigten sich polygonale, rötlich/bläuliche, teils konfluierende Papeln sowie pralle Blasen auf gerötetem Grund (Abb.1a-c). Unauffälliger Befund der einsehbaren Schleimhäute.
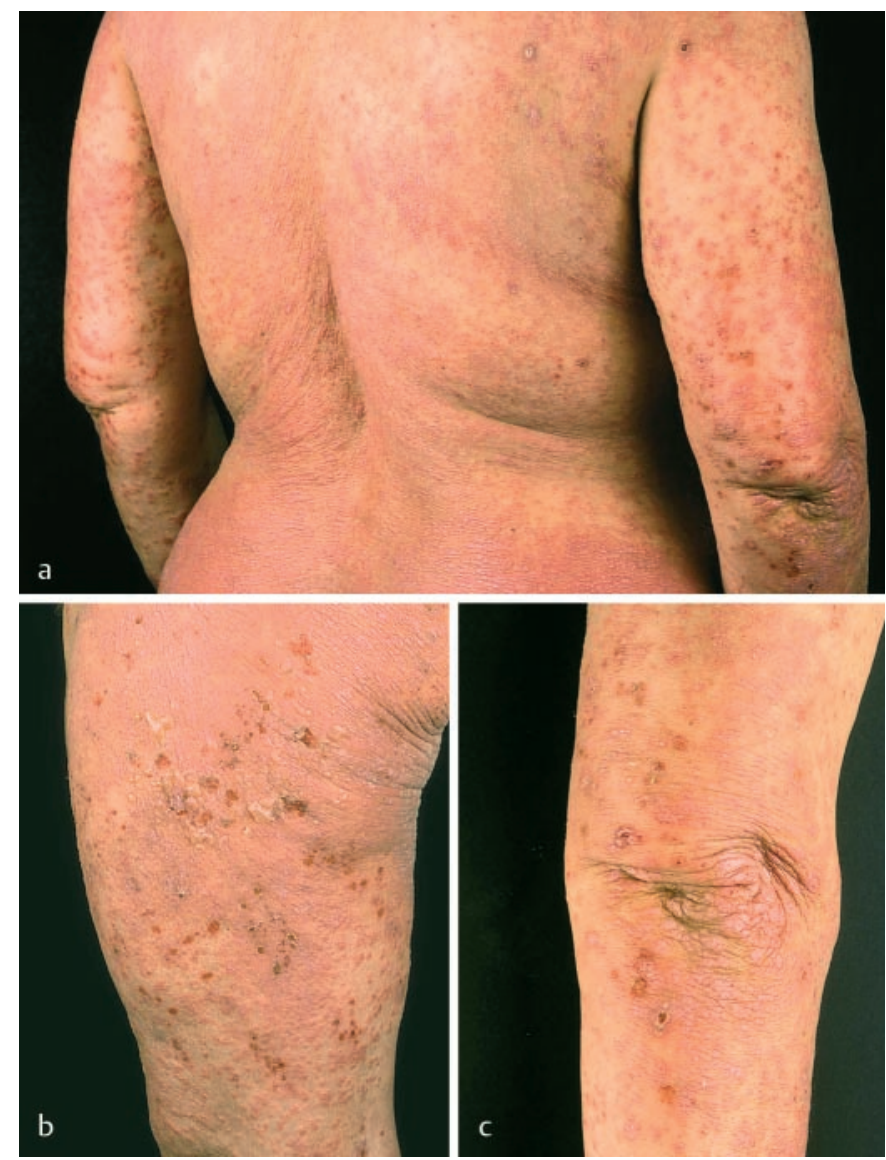

Abb. 1 Klinik eines Lichen ruber pemphigoides. a-c Polygonale, zum Teil konfluierende Papeln am gesamten Integument. b Pralle Blasen auf gerötetem Grund, vorwiegend an den Extremitäten.

\section{Befunde diagnostischer Untersuchungen Histologie}

Die Probebiopsie einer Papel vom linken Oberschenkel zeigte eine ausgeprägt akanthotisch verbreiterte Epidermis mit verbreitertem Stratum granulosum (Abb. 2a). In der aufgelockerten Basalzellage fanden sich zahlreiche nekrotische Keratinozyten, daran angrenzend ein dichtes bandförmiges, überwiegend lymphozytäres Infiltrat und Ablagerung von Melaninpigment.
In einer zweiten Biopsie von einer Blase vom Oberarm rechts stellte sich eine umschriebene subepidermale Spaltbildung dar, die von einem lockeren Fibrinnetz ausgekleidet war (Abb.2 b). Der Blasengrund war von reichlich neutrophilen und eosinophilen Granulozyten durchsetzt. Zur Tiefe hin fanden sich gemischte, teils disseminierte, teils perivaskuläre Infiltrate mit zahlreichen eosinophilen Granulozyten.

\section{Immunfluoreszenz und weitere Autoimmun-Diagnostik}

In der direkten Immunfluoreszenz konnte eine Ablagerung von Komplement C3 bandförmig entlang der Basalmembran dargestellt werden (Abb. 2c). Fibrinogen-, IgG-, IgA- oder IgM-Ablagerungen fanden sich nicht. Die indirekte Immunfluoreszenz an Normal- oder NaCL-Spalthaut war negativ. Das Serum der Patientin wurde mit Hilfe von Westernblots sowie im ELISA auf IgG-Antikörper gegen epidermale Antigene untersucht. Diese Analysen waren insgesamt negativ.

\section{Ergänzende Untersuchungen}

Laborparameter: CRP erhöht auf $28 \mathrm{mg} / \mathrm{l}$, Leukozytose von 11,28/nl, Erythrozyten 5,29/nl. Die weiteren untersuchten Laborparameter (Blutbild, Differenzialblutbild, Leberwerte, Nierenwerte, Elektrolyte, Blutfette, $\mathrm{HbA}_{1} \mathrm{C}$, Blutzucker, Gerinnung, zirkulierende Immunkomplexe, CH50, Komplement $\mathrm{C} 3$ und $\mathrm{C} 4, \mathrm{Ge}-$ samt-IgE) zeigten Normalwerte. Keine pathologischen Befunde im Röntgen-Thorax, Lymphknoten- und Oberbauchsonographie und EKG.

\section{Therapie und Verlauf}

Nach Diagnosestellung haben wir die Patientin zunächst mit hochdosierten systemischen Kortikosteroiden $(1,5 \mathrm{mg} / \mathrm{kg}$ Körpergewicht Prednisolon) über 5 Tage therapiert, bis die Blasenbildung sistierte. Dann wurde die Prednisolon-Medikation langsam, schrittweise reduziert. Hierunter kam es innerhalb von 14 Tagen zur weitgehenden Abheilung der Hautveränderungen und zu einem vollständigen Sistieren des Juckreizes. Bei einer Tagesdosis von $40 \mathrm{mg}$ Decortin $\mathrm{H}$ wurde die Patientin in die ambulante Versorgung entlassen. Im Verlauf der folgenden Monate kam es zudem zu einer nahezu vollständigen Rückbildung der Papeln, so dass die immunsuppressive Therapie weiter reduziert wurde. Nach achtmonatiger Therapie wurde die Therapie beendet, hiernach ist es nicht zum Auftreten eines Rezidivs gekommen.

\section{Diskussion}

Der Lichen ruber ist eine chronisch entzündliche Dermatose mit hoher Inzidenz ( 0,5\% der Bevölkerung). Er tritt bei beiden Geschlechtern gleichmäßig in jedem Lebensalter auf, bevorzugt jedoch ab der Lebensmitte. Bei starker Ausprägung eines Lichen ruber kann es zu einer Spaltbildung entlang der Basalmembran kommen mit klinisch sichtbarer Ausbildung von prallen Blasen und/oder Erosionen. Dies führt zur Erscheinung des erosiven Lichen rubers der Schleimhaut, oder auch (seltener) zum bullösen Lichen ruber der Körperhaut. Bei Letzterem finden sich kleine, wasserklare Bläschen in konfluierenden Läsionen eines typischen Lichen ruber. Sehr selten wird zeitgleich eine Eruption von prall gespannten Blasen auf Lichen-ruber-Läsionen und auch auf unveränderter Haut beobachtet. Diese Variante wurde 

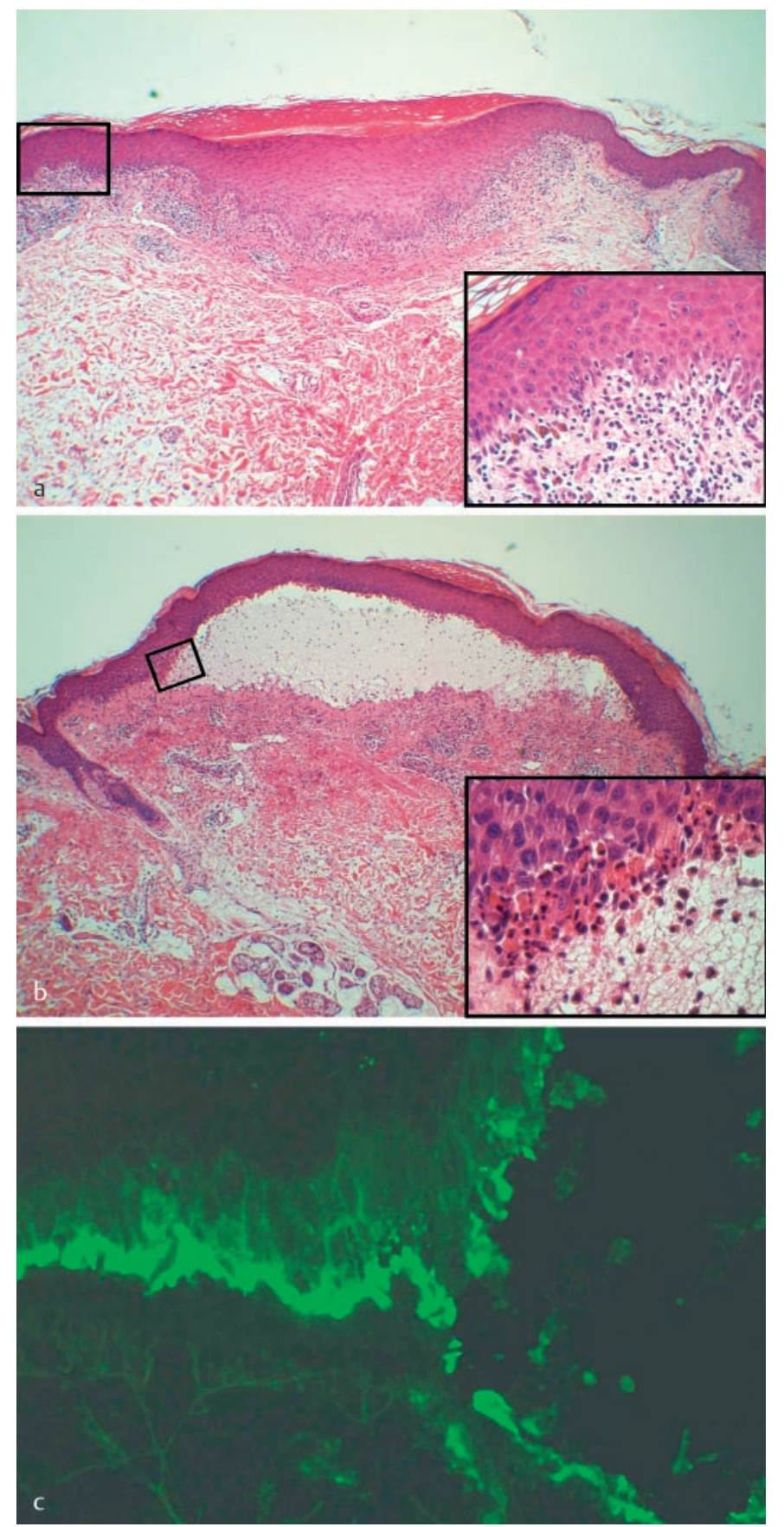

Abb. 2 Histologisches Bild des Lichen ruber pemphigoides. a PE aus papulöser Läsion (HE, $\times 20$ bzw. $\times 100)$. Ausgeprägte Akanthose, in der Basalzelllage nekrotische Keratinozyten und ein bandförmiges, lymphozytäres Infiltrat. b PE aus bullöser Läsion (HE, × 20 bzw. × 100). Subepidermale Spaltbildung, durchsetzt von neutrophilen und eosinophilen Granulozyten. c PE aus bullöser Läsion (Kryostatschnitt, × 100). Nachweis einer linearen Ablagerung von C3 entlang der Basalmembran in der direkten Immunfluoreszenz, kein Nachweis von IgG $(\times 100)$.

erstmals 1892 von Kaposi als Lichen ruber pemphigoides bezeichnet [1]. Seither sind in der Literatur nur Einzelfälle mitgeteilt worden [2-5] und die Behandlung dieser Erkrankung ist wegen der geringen Fallzahlen nicht standardisiert. Analog zur systemischen Therapie des bullösen Pemphigoids war die Therapie mit Kortikosteroiden (1-2 mg/kg KG initital) in den meisten Fällen erfolgreich.
Ursache des Lichen ruber ist eine zelluläre Autoimmunreaktion gegen die basalen Keratinozyten der Epidermis. Das entzündliche Infiltrat besteht vorwiegend aus $\mathrm{CD}^{+}$Th1-Lymphozyten und $\mathrm{CD}^{+}$zytotoxischen T-Zellen. Die fokale Zerstörung der basalen Keratinozyten über apoptotische Vorgänge mit anschließender Repopulation der Basalzellen von der Seite her führt zu einer reaktiven Hyperkeratose. Diese Hyperkeratose und das begleitende entzündliche Infiltrat führen zum makroskopischen Bild der Lichen ruber-Papel. Die Natur des Autoantigens, gegen das sich die Entzündungsreaktion richtet, ist bisher nicht geklärt. Während beim klassischen Lichen ruber die Degeneration der Basalschicht nur in der Histologie sichtbar wird, kann es bei starker Ausprägung auch klinisch zu einer Blasenbildung entlang der Basalmembran kommen. Außerdem ist im Gegensatz zum gewöhnlichen Lichen ruber eine auffällige Beimischung von eosinophilen Granulozyten zum entzündlichen Infiltrat charakteristisch für den Lichen ruber pemphigoides. Differenzialdiagnostisch abzugrenzen ist in Fällen wie diesem bei gleichzeitigem Vorhandensein von Papeln die bullöse Variante des Lichen ruber. Weiterhin könnte bei ausgeprägt polymorphen Hautveränderungen mit flächigen Erythemen, Erythema multiforme-artigen Erscheinungen und papulosquamösen Effloreszenzen klinisch auch ein paraneoplastischer Pemphigus erwogen werden.

Im Gegensatz zum klassischen Lichen ruber findet sich in der direkten Immunfluoreszenz beim Lichen ruber pemphigoides stets eine bandförmige Ablagerung von Komplement C3 entlang der Basalmembran, meist auch die von Immunglobulinen (häufig $\operatorname{IgG}$ ) entlang der Junktionszone [6]. Bei 40-50\% der Patienten sind auch zirkulierende Antikörper gegen die Basalmembranzone nachweisbar. Diese erkennen im Immunoblot Autoantigene der Größen 200 und 180 kDa (das Letztere ist identisch mit BP180) [7]. Dagegen findet man beim bullösen Pemphigoid Antikörper mit Reaktivität gegen BP180 und BP230 [8]. Vergleichbar zu Patienten mit Lichen ruber pemphigoides fanden Zillikens et al. bei Patienten mit nicht näher klassifizierbaren, subepidermalen Blasenbildungen ebenso Autoantigene von 200 kDa Größe, die genaue Erkennungsstruktur dieser Antigene ist aber bisher unbekannt [9-10]. Warum sich bei manchen Patienten, wie in unserem Fall, keine zirkulierenden Autoantikörper nachweisen lassen, ist bisher ungeklärt.

Zusammenfassend sind die diagnostischen Kriterien für den Lichen ruber pemphigoides zum einen das typische klinische Erscheinungsbild (mit Auftreten von prallen Blasen sowohl auf Lichen-ruber-typischen, konfluierenden Papeln, wie auch auf scheinbar unbefallener Haut), die charakteristische Histologie sowie die immunologischen Befunde. Blasenbildung ist beim Lichen ruber grundsätzlich ein seltenes Phänomen, differenzialdiagnostisch kommt diese als Lichen ruber bullosus im Bereich oder der Nachbarschaft typischer exsudativer Lichen ruber-Läsionen oder selten - wie im Falle unserer Patientin - als Lichen ruber pemphigoides vor. In der Literatur liegen bisher nur wenige Fallberichte über die Klinik, Diagnostik und Verlauf einer solchen Erkrankung vor. 


\section{Danksagung}

Die Autoren möchten Prof. Leena Bruckner-Tudermann und Dr. Hauke Schumann, Univ.-Hautklinik Münster, für die Durchführung der Westernblot- und ELISA-Untersuchungen danken.

\section{Literatur}

${ }^{1}$ Kaposi M. Arch Derm Syph 1892; 24: 340-341

2 Maceyko RF, Camisa C, Bergfeld WF, Valenzuela R. Oral and cutaneous lichen planus pemphigoides. J Am Acad Dermatol 1992; 27: 889-892

${ }^{3}$ Schreiner K. Lichen ruber pemphigoides und Lichen ruber vesiculosus. Arch Dermatol Syph 1930; 161: 647-657

${ }^{4}$ Hintner H, Tappeiner G, Hönigsmann H, Wolff K. Lichen planus and bullous pemphigoid. Acta Dermatologica Venereologica 1979; 85 Suppl (Stockh): $71-76$
${ }^{5}$ Richter R, Tat L. Überlegungen bei einem Fall von Lichen ruber planus mit Pemphigus vulgaris als Zweiterkrankung. Dermatol Wochenschr 1955; 131: $337-342$

${ }^{6}$ Davis AL, Bhogal BS, Whitehead P, Frith P, Murdoch ME, Leigh IM, Wojnarowska F. Lichen planus pemphigoides: its relationship to bullous pemphigoid. Brit J Dermatol 1991; 125: 263 -271

7 Tamada Y, Yokochi K, Nitta Y, Ikeya T, Hara K, Owaribe K. Lichen planus pemphigoides: Identification of $180 \mathrm{kd}$ hemidesmosome antigen. Journal of the American Academy of Dermatology 1995; 32: 883-887

8 Schmidt E, Brocker EB, Zillikens D. Neue Aspekte zur Pathogenese des bullösen Pemphigoids. Hautarzt 2000; 51: 637-645

${ }^{9}$ Zillikens D, Ishiko A, Jonkman MF, Chimanovitch I, Shimizu J, Hashimoto T, Bröcker EB. Autoantibodies in anti-p200 pemphigoid stain skin lacking laminin 5 and type VII collagen. Brit J Dermatol 2000; 143: $1043-1049$

${ }^{10}$ Kawahara Y, Matsuo Y, Hashimoto T, Nishikawa T. A case of unique subepidermal blistering disease with autoantibodies against a novel dermal 200-kD antigen. Dermatology 1998; 196: 213-216 